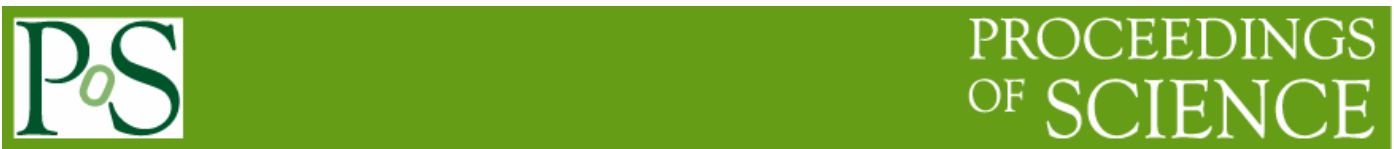

\title{
The presupernova evolution of the massive stars and the explosive nucleosynthesis
}

\author{
Alessandro Chieffi \\ Istituto Nazionale di AstroFisica - Istituto di Astrofisica Spaziale e Fisica Cosmica \\ Via del fosso del cavaliere 100, 00133Roma ( Italy) \\ E-mail: alessandro.chieffi@iasf-roma.inaf.it
}

\section{Marco Limongi}

Istituto Nazionale di AstroFisica - Osservatorio Astronomico di Roma

Via Frascati 33, 00040 Monteporzio Catone (Italy)

E-mail: marco.limongi@oa-roma.inaf .it

The key evolutionary properties of solar metallicity stars in the mass range $11 \leq \mathrm{M} / \mathrm{M}_{\mathrm{O}} \leq 120$ are briefly reviewed together to their explosive nucleosynthesis. The influence of the mass loss rate in the WNE/WCO phases on the final elemental yields is discussed in detail. We find that the elemental yields strongly depend on the size of the He core mass and that the contribution of the stars of initial mass $\mathrm{M}>35-40 \mathrm{M}_{\mathrm{O}}$ to the yields integrated over a Salpeter initial mass function does not exceed $40 \%$ (in the most favourable case) for most of the elements. Noticeable exceptions are $\mathrm{C}, \mathrm{F}$ and partly $\mathrm{N}$.

International Symposium on Nuclear Astrophysics - Nuclei in the Cosmos - IX CERN, Geneva, Switzerland

25-30 June, 2006

\footnotetext{
${ }^{*}$ Speaker
} 


\section{Introduction}

Massive stars play a major role in the evolution of the Universe since, among the other things, are responsible for the progressive chemical enrichment of the matter, eject a substantial amount of energy either as neutrinos and as kinetic energy of the ejecta, are the direct parent of the neutron stars and black holes. The "massive stars" are the stars that go through all the hydrostatic burnings, from the $\mathrm{H}$ to $\mathrm{Si}$, and then explode as core collapse supernovae. They are bordered at the lower end by the stars that develop an electron degenerate O-Ne-Mg core and at the upper end by the stars that become pair instability supernovae. The $\mathrm{H}$ and He burning phases of this class of stars have been studied in details by many researchers and problems like the extension of the convective layers, the efficiency of the mass loss and the influence of the rotation, just to mention few key physical phenomena, have been analyzed extensively. The situation is quite different as far as the advanced burning phases and the explosive burnings are concerned. Stars of mass smaller than, say, 35-40 $\mathrm{M}_{\mathrm{O}}$, have been studied by three groups, namely the one headed by Stan Woosley, a second one headed by Ken Nomoto and a third one formed by Marco Limongi and myself. The advanced phases and the explosive burnings of the stars more massive of 35-40 $\mathrm{M}_{\mathrm{O}}$ still constitute some kind of "Far West", in the sense that no paper discussing the full evolutionary properties of these models together with their final yields has been published yet. We have just computed a full set of models of solar metallicity extending between 11 and 120 solar masses and covering all the hydrostatic burnings as well as the explosive burnings. The ${ }^{26} \mathrm{Al}$ and ${ }^{60} \mathrm{Fe}$ yields produced by this new set of models have been just published in a paper devoted to the interpretation of the galactic abundances of these two $\gamma$ ray emitters [1]. In this talk I'll briefly remind a few key properties of the hydrostatic evolution of a massive star and I'll show the importance of the mass loss in the WNE/WCO phases on both the final structure of a massive star and the explosive yields.

\section{From the H burning to the Si burning}

For sake of clearness let us remind that our evolutionary code is the FRANEC (rel. 5.050419). Mass loss has been included following Vink et al. (2000) [2] in the Blue Supergiant phase and De Jager (1988) [3] in the Red Supergiant phase. The WR phase has been followed by adopting Nugis and Lamers (2000) [4], hereinafter NL00. To explore the dependence of the results on the mass loss rate in the WNE/WCO phases, we have also recomputed the evolution of the models that lose the whole $\mathrm{H}$ rich envelope, i.e. the 40, 60, 80 and $120 \mathrm{M}_{\mathrm{O}}$, with the Langer (1989) [5] mass loss formula, hereinafter LA89. The main difference between the NL00 and the LA89 mass loss rates is that the former is partly empirical and partly theoretical while the latter is mainly theoretical. We do not privilege any of the two: we simply tried both mass loss rates because they are the most used ones in stellar evolution calculations. Note that the LA89 mass loss rate is the one adopted by the Woosley group in their latest set of models.

$\mathrm{H}$ burning is characterized in these stars by very efficient losses of particles and photons from the surface and by a convective core whose mass extension determines the mass size of the forming He core. Its size is particularly important because it controls all the further evolution of 
the star. He burning is, in this respect, very similar to the $\mathrm{H}$ burning in the sense that it is also characterized by a very active

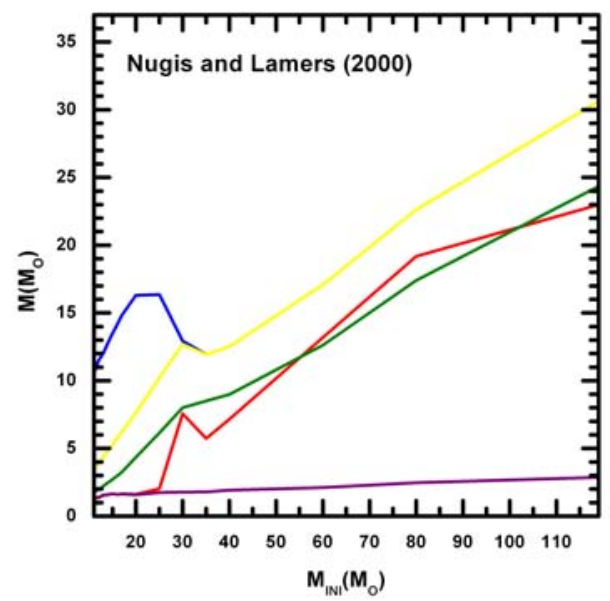

Figure 1. Key properties of the models plotted as a function of the initial mass. Outer border of the $\mathrm{H}$ rich mantle (blue); He core mass (yellow), $\mathrm{CO}$ core mass (green), Mass of the remnant for a final kinetic energy of 1 foe (red) and mass of the remnant for a fixed $\left(0.1 \mathrm{M}_{\mathrm{O}}\right)$ amount of ${ }^{56} \mathrm{Ni}$ ejected. surface from which a large amount of particles and photons escape and a convective core that is the cradle in which the CO core mass forms and that, in turn, controls the further evolution of the star. The trends with the initial mass of the total (blue or yellow), He core (yellow) and CO core (green) masses at the end of the central He burning are shown in Figure 1. Figure 2 shows the run of the same quantities when the LA89 mass loss rate is adopted. A comparison between these two figures shows that, while the adoption of the NL00 mass loss rate preserves a clear trend with the initial mass, the models computed with the LA89 mass loss rate show a quite similar final structure, and this is due to the dependence of this mass loss rate on the square of the current mass. Another important difference between these two set of models is shown in Figure 3 where the trend of the central $\mathrm{C}$ abundance at the central He exhaustion with the initial mass is shown. The blue line refers to the NL00 models while the red one refers to the LA89 models. The effect of a strong reduction of the He core mass during the central He burning is readily visible: the LA89 models end the central He burning with a much higher C Figure 2. Key properties of the models plotted as a function of the initial abundance than their NL00 mass. Outer border of the $\mathrm{H}$ rich mantle (blue); He core mass (yellow), CO counterparts. The reason is that the and mass of the remnant for a fixed $\left(0.1 \mathrm{M}_{\mathrm{O}}\right)$ amount of ${ }^{56} \mathrm{Ni}$ ejected.

models computed with the LA89 mass loss rate feel the strong reduction of the He core during 
the central He burning and hence tend to behave as stars having a similar He core mass. The total and core masses shown in Figures 1 and 2 remain essentially frozen during the advanced burning phases, $\mathrm{C}$ to $\mathrm{Si}$, since the strong neutrino losses that activate since the beginning of the central carbon burning force the nuclear burnings to speed up dramatically in order to maintain the hydrostatic evolution. To really appreciate how the two different mass loss rates, i.e. the NL00 and the LA89, affect the final structure of the stars it is very instructive to look at the different Mass - Radius (M-R) relations at the moment of the core collapse. Figure 4 shows the final $M-R$ relation for a subset of the reference

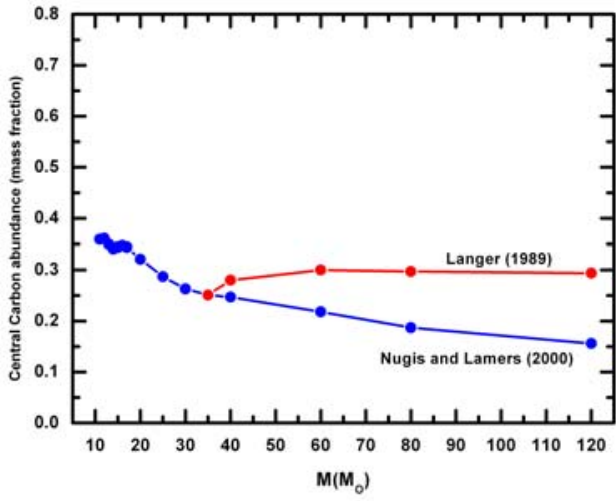

Figure 3. central $\mathrm{C}$ abundance at the end of the central He burning as a function of the initial mass. The blue and red solid lines refer to models computed in the WNE/WCO phase by adopting, respectively, the NL00 and LA89 mass loss rates.

models at the moment of the core

collapse: the larger the initial mass the more compact the structure. This result actually holds because the adoption of the NL00 mass loss rate preserves the scaling between the initial mass and the He core mass (see Figure 1). The dashed line in Figure 4 shows, vice versa, the M-R relation of the $60 \mathrm{M}_{\mathrm{O}}$ computed with the LA89 mass loss rate; the final M-R relation of this model star resembles that of a star of much smaller mass, roughly a $20 \mathrm{M}_{\mathrm{O}}$, and the reason is that its final He core mass is close to that of a star of $20 \mathrm{M}_{\mathrm{O}}$. Also the other stars, 40,80 and $120 \mathrm{M}_{\mathrm{O}}$ computed by adopting the LA89 mass loss rate show a M-R relation similar to that shown by the $20 \mathrm{M}_{\mathrm{O}}$ because also these models end up with an He core mass comparable to that produced by the $20 \mathrm{M}_{\mathrm{O}}$. The strong dependence of the final M-R relation on the final size of the He core mass has two important consequences. First of all, the amount of matter exposed to the

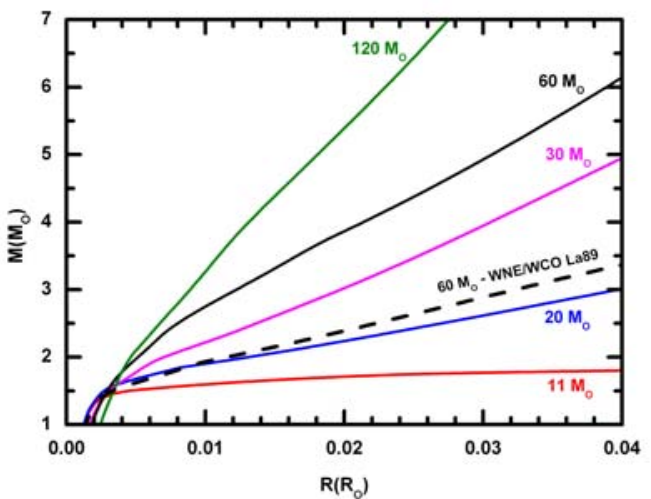

Figure 4. Mass-Radius relation for a subset of stellar models at the onset of the core collapse. The solid lines refer to the standard set while the dashed line refers to a model computed by adopting the LA89 mass loss rate in the WNE/WCO phases explosive burnings scales directly with the steepness of the M-R relation: the more compact the star the larger the amount of mass that will be exposed to the explosive burnings. Second, the binding energy of the star depends on the M-R relation in the sense that the steeper the relation the larger the binding energy and hence the larger the amount of energy necessary to eject the products of the explosive burnings. In other words, the smaller the He core mass (i.e. the larger the mass loss rate) the smaller the 
amount of matter exposed to the explosive burnings but also the smaller the energy required to eject this matter in the interstellar medium.

\section{The explosive yields}

A self consistent computation of the explosion of a massive star is still beyond the present capabilities and therefore the explosive burnings are still computed by injecting an amount of energy well within the Fe core and letting the shock wave develop and move outward on its own. Since the amount of energy injected in such a way is arbitrary, it is necessary to tune it somehow. Usually such a tuning is
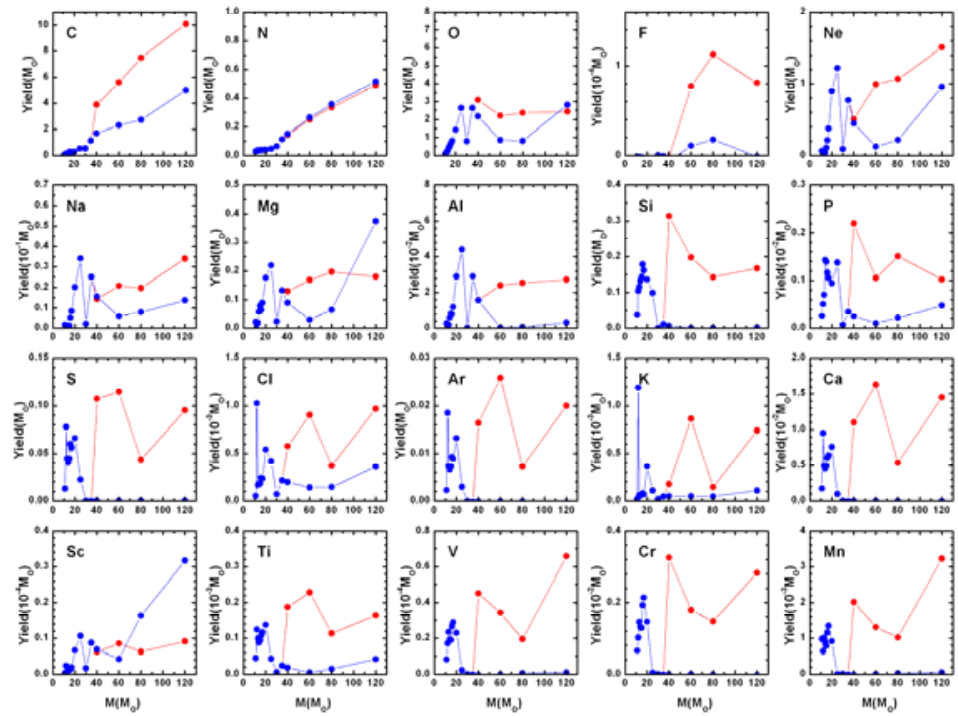

obtained by assuming

either the final kinetic energy of the ejecta or

Figure 5. Elemental yields as function of the initial mass. The blue lines refer to the standard set of models. Models that go through the WNE/WCO phases have been recomputed by adopting the LA89 mass loss rate (red lines).

the amount of ${ }^{56} \mathrm{Ni}$ to be ejected. All the properties of the explosion are fixed once the tuning is chosen, in particular the mass of the remnant. The choice of a final kinetic energy of the ejecta of 1 foe for all the masses, for example, would produce the remnant masses that are shown as a red solid line in Figure 1 for the standard models. With such a tuning, stars more massive than, say, 25-30 $\mathrm{M}_{\mathrm{O}}$, would not eject any product of the explosive burnings as well as those of the $\mathrm{C}$ convective shell. Vice versa, the choice of imposing the ejection of, e.g., $0.1 \mathrm{M}_{\mathrm{O}}$ of ${ }^{56} \mathrm{Ni}$ by each star, would have produced the remnant masses that are shown as a violet line in Figure 1. The two different tunings of the explosion do not give very different results for stars less massive than, say, $25 \mathrm{M}_{\mathrm{O}}$ in the sense that in both cases a significant amount of ${ }^{56} \mathrm{Ni}$ is ejected. Stars more massive than $25 \mathrm{M}_{\mathrm{O}}$, on the contrary, behave differently because of the steadily increase of the binding energy of the mantle with the He core mass. Hence, the ejection of at least some products of the explosive burnings, would imply in this case a quite high final kinetic energy of the ejecta. For example, the ejection of $0.1 \mathrm{M}_{\mathrm{O}}$ of ${ }^{56} \mathrm{Ni}$ from the $120 \mathrm{M}_{\mathrm{O}}$, would result in roughly 5 foes of kinetic energy in the ejecta. The behaviour of the LA89 models is quite different because their binding energy is much smaller than that of the corresponding NL00 models due to the much smaller He core masses. In this case the choice of a final kinetic energy of 1 foe already allows the ejection of a substantial amount of ${ }^{56} \mathrm{Ni}$ together to all the other products of the explosive burnings. Hence the larger the mass loss rate, the smaller the He core mass, the 
smaller the binding energy, the smaller the energy necessary to eject the products of the explosive burnings and hence the smaller the mass of the remnant. The yields obtained by assuming a final kinetic energy of the ejecta of 1 foe for the NL00 and LA89 cases are shown as, respectively, blue and red dots in Figures 5 and 6. The models having an initial mass smaller than $35 \mathrm{M}_{\mathrm{O}}$ do not differ between the two cases because they do not become Wolf-Rayet stars of the WNE/WCO kind. Figures 5 and 6 show that the NL00 models predict much larger elemental yields for the weak s component, i.e. up to the $\mathrm{N}=50$ closure shell than the corresponding LA89 models while the opposite occurs for the remaining elemental yields.

\section{The integrated yields.}

The next step to determine the contribution of the more massive stars to the global elemental yields provided by a generation of massive stars is their integration over an initial mass function. The latest results provided by Kroupa $(2003,2004)[6,7]$ seem to indicate that stars more massive than $1 \mathrm{M}_{\mathrm{O}}$ follow a quite steep power law initial mass function, the exponent $a$ being as large as 2.8 and even larger if the binarity is taken into account. In spite of this, in order to maximize the role played by the stars more massive than $35 \mathrm{M}_{\mathrm{O}}$, we show the yields integrated over a standard Salpeter $(a=2.35$ ) initial mass function (IMF). The production factors (PF) produced by a generation of massive stars ranging between 11 and $120 \mathrm{M}_{\mathrm{O}}$ are shown in Figure 7 as red dots while those corresponding to an IMF extending only up to $35 \mathrm{M}_{\mathrm{O}}$ are shown as blue dots. Obviously the two PFs have different normalization factors since each one is independently

normalized to $1 \mathrm{M}_{\mathrm{O}}$ of gas converted in mass following the chosen IMF. The close similarity between the two PFs indicates that stars more massive than $35 \mathrm{M}_{\mathrm{O}}$ do not produce any specific signature on the integrated IMF. Figure 8 shows the contribution of the more massive stars to the total yield of each element. The very low contribution of the stars more massive than 35 $\mathrm{M}_{\mathrm{O}}$ to the elements of
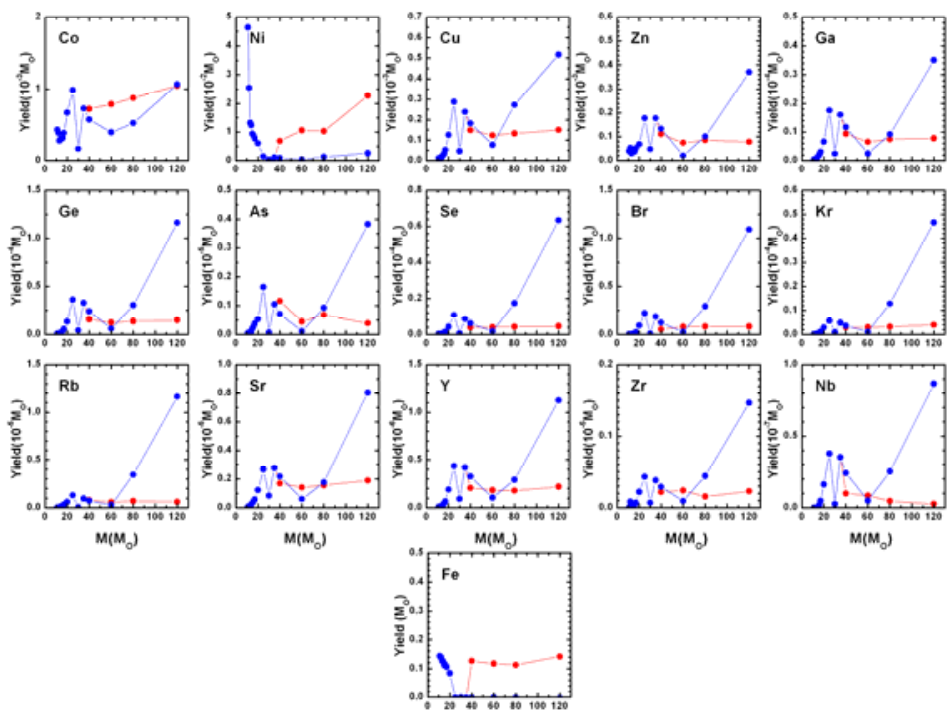

$M\left(M_{0}\right)$ $M\left(M_{0}\right)$ intermediate mass is the direct consequence of the adoption of the NL00 mass loss rate in the WNE/WCO phases and the requirement that the ejecta escape with 1 foe of kinetic energy at infinity. However, these stars contribute quite significantly to the global synthesis of $\mathrm{C}, \mathrm{N}$ and $\mathrm{F}$ because the strong mass loss 
experienced by these stars allows the ejection of these nuclei before they are destroyed by the

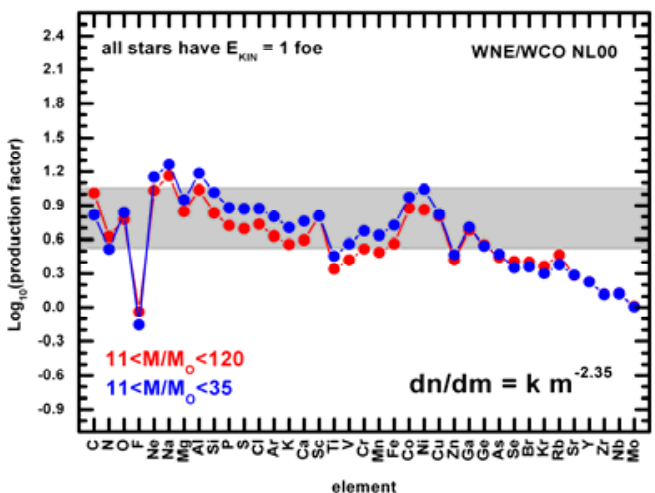

Figure 7. Production factors integrated over a Salpeter IMF. The red and blue lines refer, respectively, to an upper mass limit of 120 and 35 $\mathrm{M}_{\mathrm{O}}$. successive burnings. Also the elements mainly produced by the He burning (weak s component plus Sc) are significantly produced by these stars because a large fraction of the matter processed by the He convective core is ejected anyway in the interstellar medium in spite of the large remnant mass. The inclusion of the LA89 models above the $35 \mathrm{M}_{\mathrm{O}}$ does not modify significantly the results shown in Figure 7. However, the relative contribution of the stars more massive than $35 \mathrm{M}_{\mathrm{O}}$ to the total yields (shown in Figure 9) changes with respect to the one shown in Figure

8. In particular now these stars contribute to the total yields integrated over a Salpeter initial

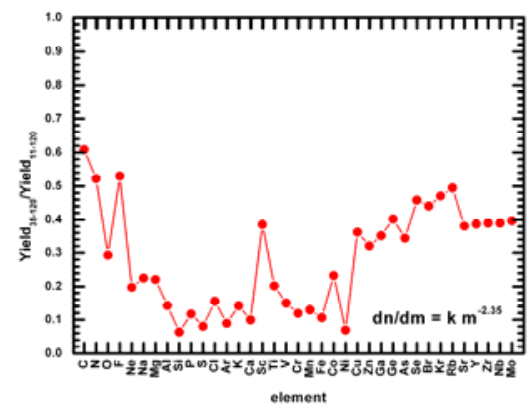

Figure 8. Contribution of the stars more massive than $35 \mathrm{M}_{\circ}$ to the total yields when the NL00 mass loss rate is adopted for the models that enter the WNE/WCO phases.

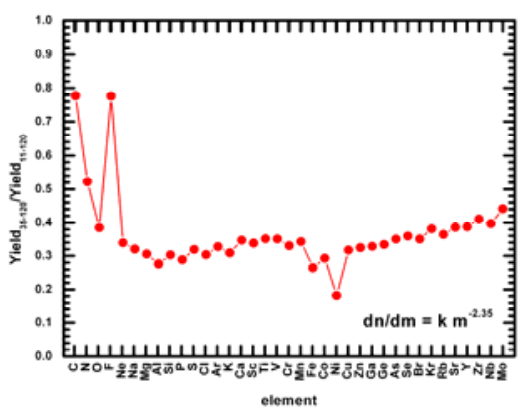

Figure 9. Contribution of the stars more massive than $35 \mathrm{M}_{\mathrm{O}}$ to the total yields when the LA89 mass loss rate is adopted for the models that enter the WNE/WCO phases

mass function by roughly $30 \%$ for most of the elements with the noticeable exceptions of $\mathrm{C}$ and F. The yields of these two elements are dominated by the more massive stars when a strong mass loss is at work. The reason is, once again, that the very efficient mass loss preserves them from the destruction.

The contributions to the total yields of the various elements provided by the stars more massive than $35 \mathrm{M}_{\mathrm{O}}$ for the two different mass loss rates in the WNE/WCO phases would reduce significantly if the IMF of these stars were much steeper than a Salpeter IMF (as suggested by, e.g., Kroupa $(2003,2004)[6,7])$.

\section{Conclusions}

We have shown that the mass loss rate in the WNE/WCO phases greatly affects the yields produced by the subset of massive stars that enter these evolutionary phase because it alters the 
final size of the He core mass that, in turn, controls the final Mass - Radius relation and hence the outcome of the explosive burnings. In particular, the smaller the He core mass, the smaller the binding energy of the structures and hence the easier the ejection of the products of the explosive burnings. The contribution of the stars more massive than $35 \mathrm{M}_{\mathrm{O}}$ to the total yields produced by a generation of massive stars depends on the adopted mass loss rate and the tuning of the explosion of a stellar model: it does not exceed $40 \%$ for most of the elemental yields (and a very favorable IMF slope). However a few specific elements like $\mathrm{C}$ and $\mathrm{F}$ may be chiefly produced by the stars that become WNE/WCO stars, especially if the mass loss is particularly efficient, because the mass loss preserves them from the destruction.

A detailed discussion of the properties of these models and of their associated nucleosynthesis will be published shortly.

\section{References}

[1] Limongi, M. \& Chieffi A. 2006, ApJ, 647, 483

[2] Vink, J.S., de Koter, A., \& Lamers, H.J.G.L.M. 2000, AA, 362, 295

[3] De Jager, C., Nieuwenhuijzen, H., \& van der Hucht, K.A. 1988, AAS, 72, 259

[4] Nugis, T. \& Lamers, H.J.G.L.M. 2000, AA, 360, 227

[5] Langer, N. 1989, AA, 220, 135

[6] Kroupa, P. \& Weidner, C. 2003, ApJ, 598, 1076

[7] Kroupa, P. 2004, New Astronomy Review, 48, 47 